\title{
El aprendizaje basado en problemas como mecanismo de formación docente: caso alumnos LEI BUAP
}

\author{
Benjamín Gutiérrez Gutierrez ${ }^{1}$ \\ tutorbenjamin@hotmail.com \\ Facultad de Lenguas de la BUAP
}

\section{RESUMEN}

El presente articulo explica como la metodología del ABP puede ser un mecanismo util en el proceso de enseñanza-aprendizaje. El ABP ofrece una gama muy amplia de investigación; se emplea en el área de Ciencias de la Salud y Medicina; no obstante actualmente es recurrente verla en las áreas de Ingenierías, Leyes, Economía(Morales y Landa, 2004), Psicología (Márquez et al., 2011) e incuso Educación (Egido et al.,2006), lo que provoca un área de oportunidad en el campo de la eduación. El objetivo del presente ariticulo es comprender la utilidad del uso del aprendizaje basado en problemas como una herramienta activa del aprendizaje significativo para desarrollar proyectos en alumnos de la asignatura de proyectos LEI-BUAP. Se encontro que la planeacion, tecnicas de enseñanza, las guias y la propia interacción procian cambios significativos en la formación de los propios sujetos.

Palabras clave: aprendizaje activo; técnicas; formación; experiencia; cambios significativos

\footnotetext{
${ }^{1}$ Profesor-investigador de la Facultad de Lenguas de la BUAP. Doctor en educación, Maestro en Educación Superior y Licenciado en la Enseñanza de Lenguas Extranjeras. Asesor en el Programa Institucional de Evaluación Académica. Pertenece al núcleo básico del Doctorado en Investigación e Innovación Educativa; es editor en el área de inglés en la revista Mexican Journal of Engineering. Es presidente y responsable del proyecto de seguimiento de egresados en la Red de Cuerpos Académicos en Lenguas Extranjeras (RECALE). «tutorbenjamin@ @otmail.com».
} 


\title{
Problem-based learning as a teacher training mechanism: LEI BUAP students case
}

\begin{abstract}
The article explains how the PBL can be a useful mechanism in the teaching and learning process. The PBL offers a wide research spectrum, it was employed in the health areas and Medicine; however, Engineering, Law and Economy are employing this methodology (Morales y Landa, 2004), Psychology (Marquez et. al.,2011) and Education (Egido et. al, 2006), which provokes an opportunity area in the education field. The goal of this article is to understand the usefulness of the PBL as an active significative learning tool to develop projects among students in the projects subject at LEI-BUAP. It was found that planning, teaching techniques, guides and the interaction promote significant changes in the training process among the subjects.
\end{abstract}

Key words: active learning, techniques, training, experience, significant changes.

Artículo recibido: 10 Setiembre. 2021 Aceptado para publicación: 15 Octubre. 2021 Correspondencia: tutorbenjamin@ hotmail.com Conflictos de Interés: Ninguna que declarar 


\section{INTRODUCCION}

La formación universitaria actual se encuentra en un estado de transición hacia un nuevo modelo de enseñanza aprendizaje que va más allá del simple hecho de la transmisión de conocimientos; este proceso implica un cambio en la concepción del proceso de aprendizaje de los estudiantes así como del cuerpo docente y de los programas educativos. Se requiere adquirir una cultura general más amplia, saber movilizar los conocimientos en la práctica, conocer y comprender al otro, emprender proyectos, así como desarrollar la autonomía y la autoestima, elementos que explica Delors (1996) en el informe, La educación es un tesoro, que realizó para la UNESCO.

Debido a lo antes mencionado y por medio de diferentes observaciones hechas en el aula y de la experiencia docente, se pude advertir que la adquisición del conocimiento no es tan difícil como resultaría ser la aplicación de dicho conocimiento dentro de diferentes contextos sobre todo de la vida cotidiana y del mundo laboral, pues lo que se pretende es desarrollar competencias para la vida. De acuerdo al Proyecto Definición y Selección de competencias (DeSeCo) de la OECD propuesto en 2011, estas se categorizan en tres áreas: Use tools interactively (utilizar herramientas de manera interactiva), Interact in heterogeneous groups (interactuar en grupos heterogéneos) y act autonomously (actuar de manera autónoma). Estas áreas de las competencias están presentes en los procesos de enseñanza aprendizaje que tienen como particularidad el ser activo.

El Aprendizaje Basado en Problemas (ABP) es una de las múltiples formas de aprendizaje activo que tiene por objetivo resolver problemas, dudas, inquietudes e incertidumbres sobre fenómenos de la vida, es decir, que se trata de un método de enseñanza centrado en el alumno con un enfoque curricular y una manera de elaborar y organizar el currículo por problemas en lugar de disciplinas que utiliza estrategias de motivación intrínseca ya que el alumno se siento motivado a encontrar la solución él solo (Egido et al,2006). El ABP cuenta con distintos elementos de instrucción que van desde el control total del problema por la parte del docente hasta la investigación dirigida y evaluada por el mismo alumno. Lo que se pretende con este método es que el alumno sea capaz de observar objetivamente, pensar de manera reflexiva y crítica y preguntarse con frecuencia ¿qué sabe sobre el tema?, ¿qué quiere o necesita averiguar? y ¿qué espera aprender? (Barell, 1999). Sánchez y Ramis (2004) dan cuenta de ello y explican que: 
"La enseñanza basada en problemas genera: (1) pensamiento crítico y capacidad para analizar y resolver complejos problemas del mundo real; (2) capacidades para encontrar, evaluar y usar apropiadamente los recursos de aprendizaje; (3) trabajar colaborativamente en equipos; (4) demostrar versatilidad y habilidades de comunicación efectiva, en forma oral y escrita; (5) usar el conocimiento y las habilidades intelectuales adquiridas en la universidad para un aprendizaje continuo". (p.103)

Todos estos elementos logran un aprendizaje más significativo, más constructivo, más social y sobre todo más comunicativo, que es lo que se pretende lograr con el estudio de este tema. Es por ello que se considera que este método se asienta principalmente de dos teorías: la teoría del aprendizaje significativo de Ausbel, y la teoría constructivista de interacción social de Vigotsky. Esta metodología tiene su nacimiento en la Universidad de Mc Master, en Canadá en 1969 (Barrows y Tamblyn, 1980). Los cursos en los que aplico por primera vez el ABP fueron los physician's medical (medicina). No obstante, este modelo se ha ido aplicando por su flexibilidad y adaptabilidad a más disciplinas, siendo el caso de las Leyes, Economía, Administración y Ciencias Culturales.

Es así que después del éxito que presentó este método, distintas universidad quisieron emular a la Universidad de Mc Master y tomaron el ABP como una nueva manera de impartir las disciplinas, donde los alumnos fueran capaces de desarrollarse plenamente y resolver cuestiones de la vida cotidiana, entre las que encontramos a la Universidad de Michigan, la universidad de New México, la universidad de Hawai y la universidad de Harvard en Estados Unidos; la Universidad de Maastrich en Holanda; la universidad de Sherbrooke en Cánada (Morales y Landa, 2004);la universidad de Acatama y la universidad de Bío-Bío en Chile (Painéan, Aliaga y Torres, 2012); así como el Instituto Tecnológico de Estudios Superiores de Monterrey y la Universidad Nacional Autónoma de México.

En el caso específico de la Benemérita Universidad Autónoma de Puebla (BUAP), en particular la Facultad de Lenguas que es donde se llevó a cabo la investigación, contempla al ABP como una herramienta de la cual se proveen algunos profesores para impartir sus clases de manera empírica y sin tener el total conocimiento de la metodología que este implica. Esto pone de manifiesto la necesidad de emprender el proyecto y dirigir la 
investigación hacia el conocimiento y buen uso del ABP para comprobar su utilidad. Cabe señalar que el aprendizaje se debe de abordar a partir de situaciones que se conozcan previamente; se habla de crear escenarios de comunicación en los que cada expresión, incluso la más sencilla, adquiera sentido y forme parte de situaciones conocidas para que se convierta en un aprendizaje significativo, apegado lo más posible a la realidad y a las situaciones de mundo laboral.

En el caso particular de esta investigación cabe destacar que el idioma inglés, ocupa un lugar importante como medio de comunicación en el mundo; ya que está presente en los cinco continentes, es también una de las lenguas oficiales de la ONU, del Comité Internacional Olímpico, de la Unión Europea, del Tribunal Penal Internacional así como la principal lengua de la Unión Africana (Cansigno, 2006).

La BUAP es una institución que se distingue por su prestigio y calidad educativa, debido a ello los objetivos de la Facultad de Lenguas es que los alumnos logren comunicarse de manera efectiva entre sus pares y con sus superiores, sean los docentes o los futuros empleadores o alumnos, por medio de la lengua extranjeras y empleándola en la solución de problemas en distintos contextos educativos. A continuación, se describe el planteamiento del problema así como la pregunta de investigación que detona al estudio y los objetivos que se pretenden alcanzar.

\section{Planteamiento del problema}

Actualmente la enseñanza activa, como se ha venido planteando, es un método más cercano a la vida práctica del estudiante, en ella se aprende haciendo y principalmente ayuda a desarrollar en el estudiante las habilidades necesarias para enfrentarse a los problemas de la vida cotidiana. Dentro de las distintas herramientas que este tipo de método ofrece encontramos el aprendizaje colaborativo, el estudio de casos y el aprendizaje basado en problemas, que es el tema que nos ocupa.

El ABP, lo define Barell (1999) como "un proceso de indagación que resuelve preguntas, curiosidades dudas e incertidumbres sobre fenómenos complejos de la vida"(p. 9), además nos ayuda a adquirir compromiso con lo que se investiga, a plantear preguntas, a investigar, a presentar informes, a trabajar en forma conjunta y a evaluar los resultados. Muchos investigadores como Barrows, et. al. (1980), McCombs (1991), Marzano (1997) y Barell (1999), han trabajado con el ABP y tiene varias razones para promoverlo, entre las que destacan: 
- Se tiene una comprensión más profunda mediante el pensamiento crítico, las estrategias de indagación y la reflexión sobre la práctica.

- Se trabaja la autodirección.

- El nivel de aprendizaje significativo logrado se da mediante el uso de información.

El ABP ofrece una gama muy amplia de investigación sin embargo, como se mencionó al inicio, en la mayoría de los casos se emplea en el área de Ciencias de la Salud y Medicina; no obstante actualmente es recurrente verla en las áreas de Ingenierías, Leyes, Economía(Morales y Landa, 2004), Psicología (Márquez et al., 2011) e incuso Educación (Egido et al.,2006), lo que provoca un área de oportunidad en el campo de la educación. Si bien el uso y aplicación de la enseñanza activa es recurrente, la mayoría del cuerpo docente y de los alumnos del área de Idiomas, que son los que competen este estudio, continúan teniendo clases tradicionales donde el proceso de enseñanza-aprendizaje es hasta cierto punto pasivo. Todo esto lo afirmo en base a observaciones, experiencias personales y al intercambio de ideas en cuanto a la impartición de clases se refiere. Muchos estudiantes pueden aprender en este entorno donde el docente habla y el estudiante escucha, lo que este tipo de clase teóricas promueven es un aprendizaje pasivo y el aprendizaje pasivo no es la forma más s/efectiva de aprender (Rosler et al., 2012). Sin embargo, con los nuevos paradigmas educativos esta concepción es cada vez más obsoleta y se tiene que trabajar más en un entorno donde el estudiante sea el que vaya construyendo sus propios conocimientos con base en experiencias; y si hablamos del aprendizaje de una lengua el trabajo interactivo es mayor.

La manera de resolver esta pasividad es por medio del constructivismo. Rosler et al. (2012) afirma que el conocimiento es creado por cada persona, con sus capacidades y habilidades, construyendo, deconstruyendo y reconstruyendo los nuevos elementos que integra a sus estructuras cognitivas; es decir, el conocimiento no se trasfiere de una persona a otra, por el contrario, debe de entenderse y apropiarse de él de manera significativa. Además, plantea resolver problemas que son de relevancia para los estudiantes. De ahí partimos para problematizar la situación en la que los estudiantes que se encuentran en clases de lenguas extranjeras deben tener clases activas en las que él propio estudiante vaya construyendo los conocimientos necesarios para su desarrollo, y que a su vez le permita resolver problemas de índole práctico y de relevancia en el contexto en el que se ve y se verá inmerso. 
Ahora bien, al hablar de los problemas que se presentan con la eficacia del ABP para desarrollar las competencias orales y cognitivas, se advierte que los alumnos de la clase de diseño de proyectos de la LEI de la BUAP, siendo el grupo afectado de esta investigación, no son capaces de desarrollar al máximo el uso de la comunicacion oral dentro del aula y mucho menos emplearla en situaciones de simulación de contextos reales. Otro de los problemas que se observan son los que se encuentran fuera del aula y van más allá del aprendizaje mismo de la lengua; estos son los problemas éticos. Por lo tanto, el objetivo de este articulo es comprender la utilidad del uso del aprendizaje basado en problemas como una herramienta activa del aprendizaje significativo para desarrollar proyectos de impacto.

\section{REFERENTES TEORICOS}

\section{Antecedentes del ABP}

En los años sesenta y principio de los setenta un grupo de educadores médicos de la Universidad de McMaster, ubicada en Canadá, se percató de la necesidad de replantear tanto los contenidos como la forma de enseñanza en la enseñanza de la medicina, "con la finalidad de conseguir una mejor preparación de sus estudiantes para satisfacer las demandas de la práctica profesional” (Morales y Landa, 2004, p. 145-146). En aquellos años la educación médica se caracterizaba principalmente por contar con clases de tipo expositivas, es decir, puramente teóricas. Luego cuando el alumno contaba con determinados conocimientos pasaban a la enseñanza clínica. Este proceso se convirtió poco a poco en "una forma inefectiva e inhumana de preparar estudiantes" (Morales y Landa 2004, p. 146). Aunado a esto estaba presente el bombardeo de información y su manejo con la entrada directa de las telecomunicaciones y el crecimiento en investigación médica.

Barrows (1996) y sus colaboradores se percatan de esta situación y configuran un nuevo método de enseñanza "en respuesta a la insatisfacción de las prácticas comunes en la educación médica" (Citado por López, 2008, p. 215). Con este nuevo enfoque la habilidad para adquirir información, sintetizarla en posibles hipótesis y probar esas hipótesis a través de la adquisición de información adicional lo denominaron "Razonamiento Hipotético Deductivo" (Morales y Landa, 2004, p. 146). Dicho proceso se basa en la antigua Grecia donde se pensaba que "el aprendizaje debería tomar lugar en situaciones concretas" (López, 2008, p.215), estas situaciones a su vez se relacionan intrínseca y 
extrínsecamente con las experiencias y conocimientos más importantes que viven los estudiantes.

En su libro Problem Based Learning: an approach to medical education, Barrows y Tamblyn (1980) dan cuenta de esto y dicen que "lerning from problems is a condition of human existence ... problem situations are learning experiences that are providing us with information and knowledge than we can apply to future problems" (p.1). Para estos autores lo más importante es usar la información, los conocimientos adquiridos y la experiencia en las actividades del día a día, ya que de esta manera se arraigan y se vuelven inolvidables. Es por todas estas características que define al ABP como "un método de aprendizaje basado en el principio de usar problemas como punto de partida para la adquisición e integración de los nuevos conocimientos" (Escribano y del Valle, 2008, p. 20).

\section{Características del ABP}

El ABP como su propio nombre lo indica, se caracteriza por plantear problemas a un grupo de personas (regularmente van de 6 a 10 integrantes por equipo) y mediante su resolución se construye paulatinamente el conocimiento, de manera tal que se investiga, analiza, resuelve y presenta la solución del problema con la ayuda de otros compañeros. Por lo tanto, este modelo busca establecer una metodología orientada a promover el desarrollo intelectual, científico, cultural y social del estudiante. Es por ello que se considera importante precisar que la innovación educativa del ABP implica un cambio significativo que involucra "la redefinición de valores y objetivos del programa académico de las instituciones, la modificación de roles del profesor y del estudiante, la transformación del proceso de enseñanza-aprendizaje y, en ocasiones, de la cultura de la institución" (López, 2008, p. 221), por lo que no es un proceso fácil de realizar y que implique una simple adecuación o actualización de contenidos, sino más bien todo lo contrario, requiere un gran esfuerzo de la parte del docente para adecuar los contenidos, de los estudiantes por modificar conductas de aprendizaje y de la institución para propiciar estos aprendizajes.

El ABP favorece en los estudiantes la capacidad para generar alternativas de solución a problemas planteados, da un espacio a los alumnos para generar conocimiento, les ayuda a reflexionar acerca de los procesos necesarios para llegar a las metas establecidas y evaluar dicho proceso para hacerle modificaciones. En cuanto a los docentes, este método 
les resulta en primer lugar desafiante, ya que rompe con las estructuras generales de una clase "tradicional" y en segundo lugar supone una preparación exhaustiva del planteamiento de los problemas a resolver y un profundo conocimiento del tema que se quiere que los alumnos trabajen en autonomía. En las palabras de Escribano y del Valle (2008) el ABP es "una técnica donde ni el contenido ni el profesor son elementos centrales" (p. 19). Pero exactamente ¿cómo funciona el ABP? Esta es una pregunta que iremos respondiendo tratando de dar las características no sólo del método, sino del medio y de los participantes; docentes y estudiantes.

\section{El método}

En el ABP es igual de importante la solución del problema como el proceso que implica el desarrollar todas las habilidades que se requieren para llegar a ella. Es por ello que es esencial que el contexto de aprendizaje se ubique en la realidad misma de los estudiantes, "en efecto, junto con la investigación académico-bibliográfica, el equipo debe realizar investigación de campo (entrevistar, encuestar, etc.), lo que significa salir del aula y conocer la realidad en que se sitúan los problemas investigados" (Paineán, Aliaga y Torres 2012, p. 163). Si bien en este caso, aunque la investigación de campo resulta casi imposible porque los estudiantes no se encuentran en un contexto social donde esté presente la lengua francesa, no significa que no puedan hacer uso de las TIC's para alcanzar los objetivos.

Un ejercicio de ABP tiene la particularidad de ser integrador, aunque no necesariamente es interdisciplinario. Esto quiere decir, que al resolver un problema se toman en cuenta los conocimientos previos que tenga el estudiante y mediante ellos se construyen los andamiajes que le servirán posteriormente para la construcción de su propio conocimiento. Un ejercicio de resolución de problemas tiene por característica el hecho de que los estudiantes "resuelven activamente el problema y aprenden durante ese proceso; los docentes son preparadores cognitivos y metacognitivos" (López, 2008, pp. 221-222).

Generalmente la problemática presentada no está estructurada y es confusa, lo que implica una restructuración cognitiva donde debe de haber una acomodación de información nueva y es por ello que a menudo cambia. Dicha información se comparte, pero el conocimiento es una construcción personal del alumno y la manera en cómo la presenta, la discusión que genera y el desafío de argumentar ponen a prueba el pensamiento. Otra 
de las distinciones que tiene el $\mathrm{ABP}$ es que la problemática no se resuelve fácilmente, al menos no con la aplicación de una fórmula específica y es por ello que, en la búsqueda de su resolución, el estudiante es quien va generando sus propios saberes, habilidades y actitudes lo que lo lleva a obtener resultados diferentes o, dicho de otro modo, nunca el resultado es una única respuesta, sin embargo, no por ello está exenta de evaluación.

De acuerdo con Sola (2005), el ABP se construye a partir de situaciones que se van presentando a los estudiantes y para hacerlo, él propone lo que se conoce como los siete pasos de ABP. El conjunto de pasos en este proceso y su ejecución hacen posible el éxito de las actividades. Si bien el proceso es largo y requiere de cierto tiempo, es fundamental cumplir con los requerimientos de cada paso, así como las exigencias que demanda su conjunto para que en verdad se dé un aprendizaje significativo y constructivo.

Presentación y lectura comprensiva del escenario.

Se presenta el escenario, la problemática. En la mayoría de los casos se trata de un texto, aunque bien puede ser un video, un audio, o cualquier otro tipo de documento. Dicho documento se debe analizar para identificar las ideas claves y los conceptos, así como aclarar aquel vocabulario que se desconozca o no se entienda. El fin es que realmente se comprenda el escenario problemática.

\section{Definición del problema.}

Tras la comprensión se debe identificar el problema y los retos que se deben de afrontar. Es importante recalcar que en este punto los estudiantes tendrán la primera impresión del problema que con el paso del tiempo se ira definiendo y redefiniendo. En este paso sólo se busca saber qué es lo que se va a resolver. Como docentes es importante saber plantear los problemas, ya que de caso contrario los estudiantes se podrían perder en el proceso y los resultados esperados no se alcanzarían.

\section{Lluvia de ideas.}

Toda vez que el problema se identificó, los estudiantes deben plantearse que es lo que deben conocer para llegar a su solución, partiendo del hecho de que él conscientemente determine que sabe y que no. Esto lo llevará a plantearse las preguntas qué, cómo, cuando, porqué, para qué, quién, etc. que a su vez generará una lluvia de ideas que conformarán una parte de un todo que se debe de buscar. Es en este momento que se empieza a gestar una hipótesis que a lo largo de los pasos siguientes madurará y se responderá. 


\section{Clasificación de ideas.}

En este momento se pretende dar orden a las ideas generadas hasta el momento. Este orden debe tomar en cuenta la relación entre una idea y otra. El resultado generalmente representa la estructura de la investigación a seguir para solucionar el problema inicial. Es importante tener claro el orden de ideas, pues de ello depende el curso que tomará la investigación.

\section{Formulación de los objetivos de aprendizaje.}

Este es uno de los momentos más importantes para el ABP, pues los alumnos deben ser muy claros y conscientes en la manera en como plantearán los objetivos ya que ellos son los que guiaran el proceso de la investigación. La redacción de estos objetivos debe de hacerse en infinitivo y deben ser lo más precisos posibles para que la acción lleve a los resultados esperados.

\section{Investigación.}

La investigación es uno de los momentos más complejos que tiene el ABP y sin embargo es mediante la investigación que se gesta el conocimiento y es la base para este tipo de método. Investigar no significa buscar fuentes de información. Es buscar hasta encontrar una intención, indagar en el lugar preciso, manejar fuentes pertinentes, hacer una comprensión de lectura eficaz para extraer conceptos claves con el fin de hacer una interpretación correcta. Investigar exige por lo tanto, contar con un plan de acción, es decir un programa de trabajo, donde se especifique la manera en la que se va a realizar la investigación.

\section{Presentación y discusión de resultados.}

Este paso es la culminación de todo el trabajo, si la presentación falla, entonces todo lo anterior falla también. Es de vital importancia que lo que se expone se argumente en una base teórica. En este punto los estudiantes presentan sus materiales al docente y comparten sus resultados con el resto del grupo de manera que la presentación en el aula debe de ser cuidadosa y desarrollar las estrategias necesarias para asegurar su eficacia. La presentación puede ser variada y dada de diferentes maneras, sin embargo debe contar con dos aspectos principales, la forma y el fondo. No sólo basta una presentación visualmente atractiva o un texto bien redactado, debe de tener un contenido bien sustentado. Se cometería un error si nos dejáramos seducir por las formas para postergar el fondo. 
A lo largo de estos pasos se desarrollan ciertos procesos cognitivos que el estudiante y el docente vinculan para que la resolución del problema ofrezca una adquisición de conocimientos y a la vez una reestructuración de aquellos que ya tenía. Escribano nos propone una manera sencilla de apreciar esto (ver figura 2.5 Procesos cognitivos implicados en el ABP), lo llama procesos cognitivos implicados en el ABP como lo podemos ver a continuación.

\section{Figura1: Procesos cognitivos implicados en el ABP}

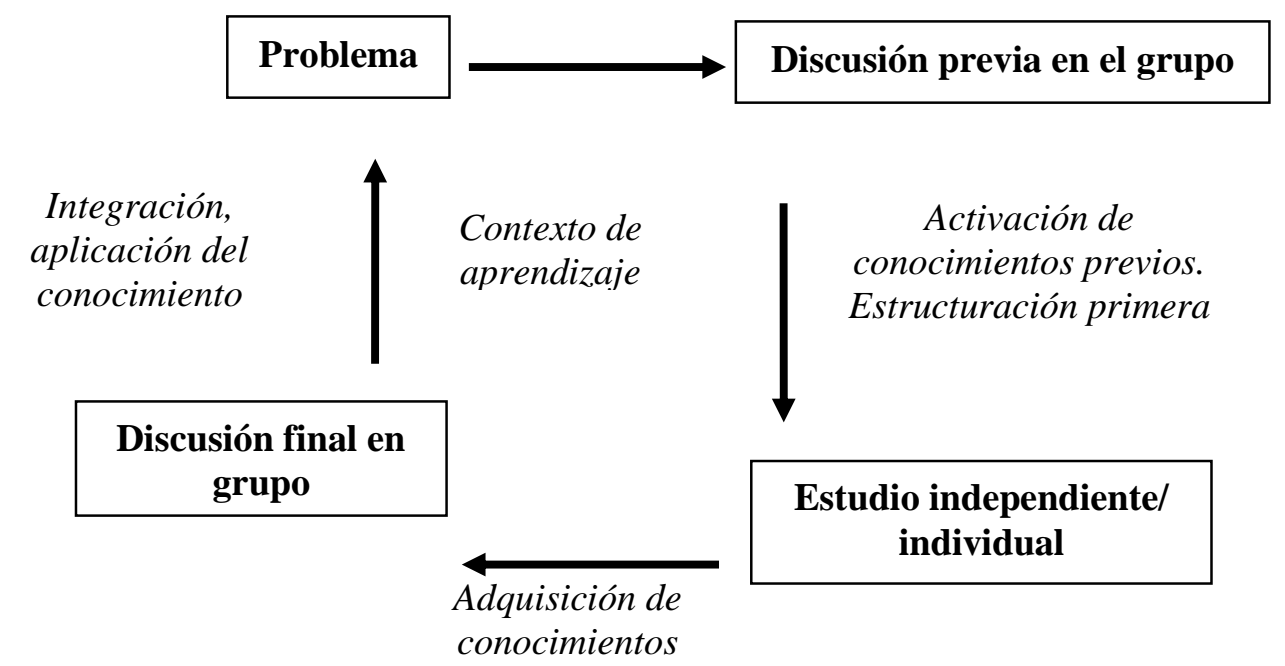

Escribano y del Valle,(coords.) (2008). El aprendizaje basado en problemas. Una propuesta metodológica en educación superior. $2^{a}$. Ed. España: Narcea. pp. 26

Como se ha visto hasta ahora, el ABP tiene muchas ventajas y produce efectos favorecedores para facilitar la comprensión de los nuevos conocimientos y promover la disposición afectiva y la motivación de los alumnos, estos dos factores son indispensables para lograr aprendizajes significativos. También ayuda a provocar conflictos cognitivos en los estudiantes que le permiten una reestructuración y equilibración de los nuevos conocimientos. En el ABP el aprendizaje resulta fundamentalmente de la colaboración y la cooperación permitiendo la actualización de la Zona de Desarrollo Próximo de los estudiantes.

Es evidente entonces que se trata de una metodología de enseñanza - aprendizaje con base constructivista que busca abordar un problema "relacionado con su futuro ámbito profesional, recogido de la realidad... con la finalidad de que durante ese proceso cada uno de los alumnos participantes pueda construir un sinnúmero de aprendizajes altamente 
significativos, pertinentes, actualizados y contextualizados" (Paineán, Aliaga y Torres 2012, p. 163). A continuación, se presentan los elementos que dan forma al ABP y hacen de él un recurso didáctico pedagógico completo. En primer lugar, presentamos el medio o el ambiente que se debe propiciar para su éxito, el rol del docente, el rol del alumno.

\section{El medio}

Al hablar de la enseñanza aprendizaje se suele pensar exclusivamente en la relación que existe entre el docente y el estudiante. Sin embargo, el medio o el ambiente en donde se desarrolló este proceso y en donde ocurren las interacciones también pueden propiciar tanto un buen como un mal aprendizaje, es por esta razón que se debe tomar en cuenta para que se favorezcan las relaciones interpersonales, no sólo entre estudiantes sino también entre estos y el docente. Generalmente el aula es el escenario donde sucede todo, o casi todo. El aula debe convertirse entonces en el medio donde prevalezca la confianza, la comunicación abierta y la disposición a tomar riesgos para enfrentar los problemas. Cuando se trabaja con el ABP, es importante que el aula, no sólo sea una habitación con sillas de trabajo, un escritorio y un pizarrón, eso es simple infraestructura que apoya la labor docente y el aprendizaje de los estudiantes; también es necesario que esas cuatro paredes se transformen en un medio acogedor que invite a los estudiantes a participar haciendo preguntas, a investigar para encontrar respuestas, a crear relaciones significativas y a reflexionar sobre la manera en cómo aprende. El ABP cuenta con un medio investigativo que necesita para desarrollar valores, creencias, conductas y actitudes. Sin importar cuales sean estas, el ambiente debe contar siempre con determinados elementos que propicien la investigación y a su vez el aprendizaje. Barell (2006) en su obra Aprendizaje Basado en problemas: un enfoque investigativo, propone seis:

\section{El docente como modelo}

Un modelo es alguien o algo que representa una conducta, relaciones o roles que son ejemplares. Siguiendo esta línea el docente debe ser un modelo en el cual los estudiantes vean reflejado la manera de enfrentar situaciones problemáticas. Barell cree que cuando un docente comparte con sus estudiantes experiencias de éxito o fracaso, les hace saber que nadie es perfecto, que los docentes no tienen todas las respuestas y que son vulnerables. Esto propicia que se establezca una asociación donde los docentes y los estudiantes comparten cosas en común. 


\section{Interrogación}

A lo largo del ABP se tienen que ir desarrollando preguntas a las cuales se les tienen que dar respuesta, de hecho, la problemática se presenta de esta manera, como un medio para investigar y así determinar la mejor solución. Las preguntas pueden ser diversas, pueden presentarse de manera simple o de manera compleja. Lo importante es que las situaciones problemáticas llenas de dudas, dificultades, ambigüedades y cuestiones sin resolver puedan servir como punto de partida para la identificación de problemas. La mejor manera de hacer es mediante un marco interrogativo. Barell (2006) nos propone el que está basado en el Intelecto de Tres Niveles. Este modelo ofrece a los docentes una manera de pensar o dar cuenta como aprenden los estudiantes a comprender de forma profunda y significativa.

El Intelecto de Tres Niveles tiene tres metas; retener, comprender y aplicar la información. Cada una de ellas utiliza diferentes verbos que representan las exigencias intelectuales. Para el retener se hacen preguntas de primer nivel que implique reunir información, para ello se vale de utilizar los verbos ubicar, describir, investigar, buscar, hablar sobre el tema, identificar, seleccionar, recordar. Para el comprender se hacen preguntas de segundo nivel en las que encontramos la manera de procesar la información, las preguntas que se plantean deben contener los verbos razonar, comparar, contrastar, separar, resolver, distinguir, explicar, clasificar, analizar, inferir. Finalmente, para el aplicar se hacen preguntas de tercer nivel que contengan los verbos evaluar, imaginar, juzgar, predecir, especular, aplicar un principio, estimar, anticipar. Como se puede ver, cada pregunta requiere de quienes las contesten un nivel diferente de desafío intelectual que se presenta de manera progresiva yendo de lo más simple a lo más complejo, de lo concreto a lo abstracto.

\section{Calidad de respuestas del docente}

Es importante que para propiciar un ambiente de confianza y empatía el docente procure dar respuestas y comentarios a los estudiantes, y la manera en cómo lo haga, muchas veces es incluso más importante que la pregunta en sí misma -como en la filosofía, lo importante no es la pregunta sino la respuesta que se da-. En un estudio realizado por Siegel en 1990 sobre las habilidades intelectuales concluyó que "el tono de voz, la forma como atiende a lo que los alumnos dicen y el interés del docente en las ideas de los alumnos podrían comunicar muchas cosas que contribuirían a crear un ambiente acogedor 
y favorable" (Siegel, 1990, citado por Barell, 2006, p. 44). Por ello es importante que el docente de respuestas que impliquen una empatía con los sentimientos, que solicite a los estudiantes las razones de dicha pregunta, que invite a construir más a fondo la idea o que ofrezca más información, que de claridad o pida dar claridad al tema, que establezca relación con las respuestas de otros estudiantes y que siembre en el estudiante la reflexión metacognitiva. El objetivo de dar respuesta es comunicar un interés genuino en saber más sobre lo que piensa y lo que siente el estudiante.

\section{Interacción entre pares y Desarrollo de habilidades de investigación grupales}

En el ABP no solo se trata de preguntas y respuestas. Si bien hasta el momento sólo se ha hablado de ello, no quiere decir que para la pregunta sólo exista una clase de respuesta ya que precisamente la resolución de una problemática puede darse de distintas maneras, con excepción de las ciencias duras. La intención del ABP es buscar la que mejor se adapte a los contextos y para ello se trabaja colaborativamente. Esto implica que exista un intercambio de ideas donde cada integrante del equipo trata de llegar a su propia conclusión basada en evidencias, que quizás puede llegar a ser la respuesta general, mientras escuchan una gran diversidad de puntos de vista. Por lo tanto, las habilidades para la investigación en equipo o grupales se logran escuchando, concentrándose en el tema, construyendo conocimientos sobre las ideas propias y las de los otros.

\section{Usar diarios de reflexión}

Barell (2006) sugiere el uso de un diario de reflexión cuya finalidad es la reflexión metacognitiva, es decir el qué, el cómo y el para qué se aprende algo, dicho de otro modo, la reflexión sobre las actividades es lo que las convierte en experiencias significativas. Según este autor poner de manifiesto estas reflexiones puede contribuir de manera significativa el desarrollo no sólo del intelecto y la manera de aprender, sino también la manera de relacionarnos con los demás (Barell, 2006). Aunque sea un trabajo individual, es importante tomar en cuenta que a las conclusiones a las que llegan los alumnos están basadas en el intercambio de ideas y la interacción con sus pares y el docente.

En suma, podemos decir que la actuación de los docentes como modelos, hacer distintos tipos de preguntas, dar respuestas de calidad, generar interacción y reflexionar sobre los aprendizajes animan a los estudiantes a contribuir, a hacer preguntas no convencionales, a contradecir y sobre todo a generar un ambiente agradable, respetuoso y acogedor para el ABP. 


\section{El docente}

La manera de proceder de los docentes es la de actuar como guías en el proceso de construcción del conocimiento. La manera en cómo presentan el material y la forma en cómo se desempeñan en este proceso “debe adecuarse a las condiciones del grupo, orientar la investigación y estimular el pensamiento del equipo a través de preguntas que iluminen las vías de acceso" (Paineán, Aliaga y Torres, 2012, p. 163). Pareciera que a lo largo del procedimiento que encierra los sietes pasos, los cuales se presentaron anteriormente, el docente debe estar presente pero no de manera protagonista, sino todo lo contrario, debe jugar un papel pasivo, donde sus intervenciones solo sirvan de acompañamiento para la construcción de saberes de los estudiantes. Sin embargo, esto entendido está un poco fuera de proporción. Si bien es cierto que el aprendizaje activo reclama la participación constante de los estudiantes no quiere decir de ninguna manera que el docente deje de existir.

En un método como el ABP el docente tiene un rol importante a desempeñar, Sola (2005) suele decir que se trata de un "director de orquesta" (p. 43), este trabajo lo lleva a tener claro los objetivos, el tema, y cómo es que los estudiantes van a llegar a aprender a aprender, aprender a hacer, aprender a vivir juntos y aprender a ser. No se trata sólo de escoger al azar un documento, plantear un problema y dejar a la deriva a los estudiantes. El docente es quien debe asegurar que el camino que se está navegando sea el apropiado y de no ser así, debe de corregir el rumbo cual timón; y los objetivos se convierten en la carta de navegación que los estudiantes deben de ir interpretando para que la llegada a puerto, al conocimiento, sea asequible. Y esta es una de las razones por las que la relación entre el docente y los estudiantes no está separada, desligada; se trata más bien de una relación estrecha y de confianza. Por su parte, comenta López (2008), que los docentes se vuelven "sus colegas en la resolución del problema, modelos del interés y el entusiasmo por aprender, y además preparadores cognitivos que fomentan la creación de una atmósfera en la que se promueve una indagación abierta" (p. 220).

En el ABP los docentes son quienes plantean problemas del mundo real y el juego de roles, mientras respaldan el aprendizaje "poniendo a prueba, cuestionando y desafiando el pensamiento de los estudiantes" (López, 2008, p. 219). Sin embargo, esto no sería posible si el docente no conociera el tema. Es necesario entonces que el profesor sea un 
experto para que la guía y el acompañamiento que les ofrecerá a los estudiantes sea de calidad, y por ende el alcance de los objetivos se logre.

Como lo menciona Sola (2005), el docente "además de dominar su disciplina debe programar sus materias y pensar hasta el último detalle en lo que se va a hacer dentro y fuera del aula" (p. 39) así pues no sólo se debe preguntar que van a aprender los estudiantes, sino cómo lo van a hacer de tal manera que el aprendizaje memorístico se torna un aprendizaje significativo y activo. Es imprescindible entonces, que el docente pase de ser un especialista que sabe dar y explicar un tema, a ser un docente "profesional del aprendizaje" (Escribano y del Valle, 2008, p.25) que hace todo lo que está en sus manos para facilitar el acceso cognitivo de los alumnos a los contenidos y a las prácticas profesionales que el problema abarca.

Para que el aprendizaje sea significativo, autorregulado, autónomo; el docente no sólo tiene que pensar en los contenidos, sino también en quienes son los que lo van a aprender. En este sentido, el docente debe realizar un valioso trabajo de preparación, de diseño de instrucciones claras y comprensibles, estableciendo en todo momento una articulación oportuna entre los distintos temas de estudio. Todo esto lo debe llevar a plantearse como abordar el problema, cuáles son las dificultades con las que se van a encontrar los alumnos, como facilitar los materiales para un crecimiento intelectual, que ayudas complementarias les servirán a los estudiantes para que su progreso sea autónomo, y lo más importante tiene que preguntarse como aprenden los estudiantes, para que de esta manera se favorezca una didáctica centrada en el aprendizaje. Dicho de otro modo, el docente tiene que conseguir que el estudiante "estudie con la mayor independencia posible y, que, en este proceso, él está como una fuente de información más" (Escribano y del Valle, 2008, p. 27).

Otra de las cualidades que debe tener un docente que trabaja con el ABP es la habilidad que tenga para supervisar el proceso garantizando la participación de cada miembro del grupo de forma colaborativa. Recordemos que el ABP se basa justamente en como los equipos trabajan para alcanzar metas en común y dicha meta es la resolución de problemas. Es indispensable, pues, que el docente cuente con las estrategias adecuadas para "favorecer el proceso de análisis, comprensión y estudio del problema" (Escribano y del Valle, 2008, p. 28) así como estimular el trabajo colaborativo y dejar de ser el centro de atención y la fuente primera de información. Su participación no desaparece, aunque 
no es ciertamente el actor principal. Su participación debe "ser atinada y sutil" (Sola, 2005, p. 53) de tal manera que les permita a los estudiantes cierta independencia y su atención permanezca a lo largo del proceso.

\section{Los estudiantes}

El ABP exige en los estudiantes un trabajo autónomo, donde él mismo es el mayor responsable de la construcción de su conocimiento, adquiere un rol activo en el cual es protagonista de los escenarios y es quien, con el acompañamiento del docente, hace de los aprendizajes algo significativo. El estudiante aprende a "determinar qué necesita conocer" (López, 2008, p. 216) es decir que aprende a aprender adaptándose al nuevo conocimiento, a los desafíos y a los problemas que se le presentan. En el ABP se espera que eventualmente el estudiante tome responsabilidad de su propio aprendizaje, es decir, que se convierta en el protagonista principal del proceso. Sola (2005) menciona que "su tarea es salir al encuentro del conocimiento con la ayuda de herramientas metodológicas y del profesor para lograrlo con eficiencia" (p. 46). De esta manera, son los propios estudiantes quienes llegan a ser "comprometidos creadores de soluciones que identifican la raíz del problema y determinan las condiciones necesarias para llegar a una buena solución” (López, 2008, p. 220), entonces resulta que siempre estén buscando el sentido y la comprensión, que se terminan convirtiendo en los elementos que dirigen su propio aprendizaje.

Por su parte, en su forma colectiva, el ABP estable un amplio margen de iniciativa a los grupos para afrontar la solución del problema planteado. Generalmente cuando se presentan resultados se da cuenta de un trabajo investigativo muy concreto, que incluye propuestas de actuación adecuadas y refleja los conocimientos y las áreas sobre los que normalmente se trabaja. Con la ayuda de este recurso pedagógico, los alumnos llegan a plantear fundamentos, registros e incluso actividades específicas; encajando con buen criterio las diferentes aportaciones de los miembros del grupo (Egido et al., 2006).

A lo largo del proceso de resolución de problemas, el estudiante trabaja en pequeños equipos, conjuntando habilidades colectivas de adquisición, comunicación e integración de información. Todo este conjunto de elementos le van a permitir (López, 2008):

- Pensar críticamente y poder analizar y resolver problemas reales y complejos.

- Identificar, evaluar y utilizar recursos apropiados de aprendizaje.

- Trabajar de manera cooperativa en equipos pequeños. 
- Demostrar efectivas habilidades de comunicación oral y escrita.

- Usar habilidades intelectuales y de conocimiento adquiridas en la universidad, para lograr el aprendizaje continuo.

En suma, entender el papel que se le otorga al estudiante y asumir el compromiso de un aprendizaje autónomo basado en la acción es una de las primeras exigencias del ABP, ya que el acercamiento al conocimiento demanda que cada persona se comprometa con ella misma y se responsabilice e implique en crear su propio saber; por ende, el énfasis que se pone en el aprendizaje auto-dirigido requiere esfuerzo y una actitud activa pues el aprendizaje depende del estudiante mismo y no del profesor.

\section{METODOLOGIA}

Esta investigación se realizao en la clase de diseño de proyectos, la cual se imparte en los primeros semenstres de la licenciatura. Se les solicito que los alumnos que conformaran equipos y que realizaran un proyecto con impacto social. Los estudiantes realizaron su proyecto en escuelas, casas de retiro, orfanatos y casas de cultura.

Una vez terminado y evaluado los proyectos se procedio a documentar el caso de los almnos de la asignatura de diseño de proyectos. El enfoque que se siguio fue de tipo cualitativo el cual intenta entender el fenomeno estudiado. La recolección de la informacón se realizo de tipo transversal, mediante la tecnica de grupo focal y utilizando una entrevista estructurada de 15 preguntas abiertas.

Los participantes fueron 17 alumnos, 10 mujeres y 7 hombres, que al momento estan registrados en la asignatura.

\section{RESULTADOS}

Una de las preguntas que se le hizo a los participantes, estaba diseñada para cuestionar sobre la utlidad de las guias para diseños de proyectos. Esta guias fueron diseñadas por el autor y ya han sido probadas en diferentes contextos, generando productos de ivestigación previos. A continuación se presenta los resultados para su análisis. 
Tabla 1: Utilidad de guias para el diseño de proyectos

\begin{tabular}{ll} 
CODIGO & \multicolumn{1}{c}{ RESPUESTA } \\
\hline P1S1 & $\begin{array}{l}\text { Fue muy alto ya que las actividades llevaban una secuencia lógica ya que } \\
\text { las actividades eran secuenciadas }\end{array}$ \\
\hline P1S2 & $\begin{array}{l}\text { Las actividades fueron eficaz y eficiente pues en cada persona hubo un } \\
\text { cambio y cada uno aprendió de manera unitaria }\end{array}$ \\
\hline P1S3 & $\begin{array}{l}\text { Fue alto ya que aprendimos hacer cosas que no se habían hecho antes, y } \\
\text { desarrollamos un proyecto y al tener una guía de secuencias fue más fácil } \\
\text { realizarlo }\end{array}$ \\
\hline P1S3 & $\begin{array}{l}\text { Fue muy eficaz ya que todas las actividades fueron de manera progresiva } \\
\text { y eso permitió culminar con el proyecto y a la vez permitió generar } \\
\text { conocimiento por nosotros mismos }\end{array}$ \\
\hline
\end{tabular}

$\overline{\text { Evidentemente los sujetos consideran que el contar con un guía para la creación de }}$ proyectos, les permite controlar sus acciones y al mismo tiempo darles orden lógico, logrando que el trabajo sea significativo, tal y como lo plantea Barell (2001).

En el mismo tenor, se les cuestiono sobre la técnica de lluvia de ideas como proceso de genración del proyecto a realizar. Esta pregunta tuvo la intención de forzar a los participantes a reflexionar sobre el proceso que siguieron para la creación de sus proyectos. Vesase los datos para su análisis.

\section{Tabla 2: Utilidad de la lluia de ideas en la generacion de un proyecto}

\begin{tabular}{ll} 
CODIGO & \multicolumn{1}{c}{ RESPUESTA } \\
\hline P2S5 & $\begin{array}{l}\text { En mi opinión es de gran ayuda, ya que nosotros cuando estábamos } \\
\text { haciendo el proyecto tuvimos que tener cuidado en el uso de conceptos y } \\
\text { llegar a un común acuerdo para plasmarlos en el cuadro de metas y si es } \\
\text { de gran ayuda }\end{array}$ \\
\hline P2S3 & $\begin{array}{l}\text { Fue una técnica muy adecuada ya que de esa forma pudimos ver cómo, } \\
\text { aunque sabemos algunos términos, otros compañeros tenían otro y eso } \\
\text { permitió sacar más provecho }\end{array}$ \\
\hline P2S6 & $\begin{array}{l}\text { Utilizarla de manera personal o en grupo proporciona muchos beneficios } \\
\text { ya que te permite aportar y que otros lo hagan y de esa manera el trabajo } \\
\text { es más rápido }\end{array}$ \\
\hline P2S7 & $\begin{array}{l}\text { Es buena ya que pone a prueba lo que sabes para que seas capaz de aceptar } \\
\text { otra idea diferente a la tuya }\end{array}$ \\
\hline
\end{tabular}

Es importante recalcar que en este punto los estudiantes tuvieron la primera impresión del problema que con el paso del tiempo se fue definiendo y redefiniendo como un mecanismo de reflexión. Evidentemente, como futuros docentes es importante saber plantear los problemas, ya que de caso contrario los estudiantes se podrían perder en el 
proceso y los resultados esperados no se alcanzarían confirmando lo que Sola (2005) menciona respecto a la lluvia de ideas como mecanismo de aprendizaje activo.

El siguiente item tuvo la finalidad de comprender el nivel de planeacion que ellos tuvieron en la creación de un proyecto. Este reactivo en la guia de entrevista es fundamental en la para entender como aplicación del ABP, puede benefiar el proceso de formación. La tabla 3 muestra los resultados obtenidos.

\section{Tabla 3: Nivel de planeación para la elaboración de un proyecto}

\begin{tabular}{ll} 
CODIGO & \multicolumn{1}{c}{ RESPUESTA } \\
\hline P8 S4 & $\begin{array}{l}\text { Hacer un proyecto no es de un día para otro es laborioso y lleva un proceso } \\
\text { largo para que realmete se pueda alcanzar las metas. }\end{array}$ \\
\hline P8S6 & $\begin{array}{l}\text { Cuando ya tienes un modelo o libro es fácil, pero en nuestro caso fue } \\
\text { pensar y quebrarnos la cabeza para planear y elegir materiales aptos para } \\
\text { niños. }\end{array}$ \\
\hline P8S14 & $\begin{array}{l}\text { Creo que es necesario tener un modelo de placeación que te permita seguir } \\
\text { pasos para poder alcanzar metas y eso es importante. }\end{array}$ \\
\hline
\end{tabular}

Evidentemente el ABP proporciona los mecanismos necesarios no solo para planear, sino para ejecutar y valorar las diferentes etapas del proyecto. Aunque los participantes lo ven laborioso por el propio proceso en si, favorece el proceso de análisis, reflexión y crítica para comprender y realizar interveniones más acertivas; tal y como lo menciona López (2012) y Escribano y del Valle (2008).

En este apartado se procedio a procedio a terminar la utilidad de los elementos ABP en el diseño de proyectos mediante el conteo de frecuecias. Recordemos que el metodología ABP se compone por ocho elementos escenciales que son: método, presentación de esenario, problema, lluvia de ideas, clasificación, formulación de propuestas, investigación y resultados. Vease tabla de frecuencias 4.

Tabla 4: Frecuencias de la utilidad del ABP

\begin{tabular}{|l|c|c|c|}
\hline \multicolumn{1}{|c|}{ Categoría } & Muy util & Poco util & Nada util \\
\hline Método & 15 & 2 & 0 \\
\hline Presentación del esenario & 8 & 7 & 2 \\
\hline Problema & 9 & 5 & 3 \\
\hline Lluvia de ideas & 7 & 9 & 1 \\
\hline Clasificación & 6 & 8 & 3 \\
\hline Formulación de propuestas & 8 & 6 & 3 \\
\hline Investigación & 9 & 6 & 2 \\
\hline Resultados & 10 & 5 & 2 \\
\hline
\end{tabular}


Evidentemente, los alumnos reconocen que el metodo del ABP, el analisis que se emplea del contexto a intervenir, la determinación del problema que debe ser observable y medible, la formulación de la propuesta concensuada, la intervención investigativa y el reporte de resultados conforman un contructo fuerte en el proceso de formación y la elaboración del mismo permitiendo fomentar el pensamiento croitico, analitico y reflexico mediante procesos de hetero, co-evluacion y autoevaluacion constante. La lluvia de idas y la clasificacion de las mismas parecieran ser un obstaculo; sin embargo, son procesos necesarios en la maduracion del propio sujeto el cual tiene que proponer, negociar y aceptar nuevos puntos de vista en vias mejorar la propia intervension.

Otra de las preguntas en la guía de entrevista se encamino a explorar aquellas cuestiones emocionales que los paticipantes enfrentaron durante su proceso de intervensión. Evidentemente, el ABP es un método que tiene como eje medular la provocación de un cambio y no puede existir ningun cambio sino pasa por el filtro emocional. La siguite tabla muestra los comentarios obtenidos.

\section{Tabla 5: Comentarios que provoco situaición emocional}

\begin{tabular}{|c|c|}
\hline CODIGO & RESPUESTA \\
\hline P12S14 & $\begin{array}{l}\text { En la sesion de happy birthday Rodrigo, pregunto a quien le gustaba su } \\
\text { cumpleaños y una niña menciono que no le gustaba porque su familia lo arruinaba } \\
\text { todo ya que siempre peleaban y eran disfuncionales. Nos causo conflicto porque } \\
\text { no sabiamos cómo reaccionar y provoco incomodidad y todos tuvimos prolemas } \\
\text { para hacer algo. }\end{array}$ \\
\hline P12S15 & $\begin{array}{l}\text { El utlimo día que realizamos nuestra actividad un niño se acerco a mi y me abrazo } \\
\text { y me dijo que si regresaríamos al siguiente día, le pregunte por qué y me comento } \\
\text { que porque sentia que él era imporatante par nosotros, se me hizo un nudo en la } \\
\text { garganta y no supe que decir. }\end{array}$ \\
\hline P12S12 & $\begin{array}{l}\text { En la última sesión nos recibieron los niños y nos dijeron que por qué no } \\
\text { hablamos llegado la sesión pasada (la madre había suspendido clase). Nosotros } \\
\text { les dijimos que no habíamos podido y ellos preguntaron si regresaríamos, a lo } \\
\text { que respondimos que si y ellos nos pidieron que solicitáramos niños de 6to ya } \\
\text { que ellos pasarían a ese grado. }\end{array}$ \\
\hline P12S2 & $\begin{array}{l}\text { Un niño nos dijo que él no quería estar ahí y eso me hizo pensar que estamos } \\
\text { haciendo mal por qué no quiere tomar la sesión. }\end{array}$ \\
\hline P12S1 & $\begin{array}{l}\text { Hubo un niño que a todos nos soprendió ya que me causo alegria proque no había } \\
\text { hecho su propio dispensador que había colocado a fuera de su casa y eso me hizo } \\
\text { pensar que si había funcionado el proyecto. }\end{array}$ \\
\hline P12S11 & $\begin{array}{l}\text { Un señor nos dijo que si le habia gustado nuestra clase y que si le había entendido } \\
\text { eso nos hizo sentir bien y luengo nos enteramos que él había sido profesor de } \\
\text { inglés. }\end{array}$ \\
\hline
\end{tabular}


Los alumnos están claros y consciente no solo en la manera en como plantearon los ojetivos, ya que ellos son lo que guiaron el proceso de la investigacion; sino también, como se afrontan las situaciones emocionales. Lo que favorecio plenamente e proceso de formación en integracion con el contexto, como lo ha expresado Sola (2005) y Paineán, Aliaga y Torres (2012) que mencionan que todo proceso formativo implica la vinculacion de cuestiones extrinsecas e intrinsecas directamente relacionadas que tienen como finalidad provocar cambios visibles en los sujetos.

Fianlmente, se les pidio que dieran recomendaciones para la mejora del proceso. Este reactivo tiene tuvo como finalidad generar una retroalimentación que favoresca el proceso. No puede existir ningun proceso formativo que no tenga una hetero evaluación

Tabla 6: Recomendaciones

\begin{tabular}{l|l} 
CODIGO & \multicolumn{1}{c}{ RESPUESTA } \\
\hline P15S14 & $\begin{array}{l}\text { Me gusto que la case no fueran tan teórica, pero creo que hacer más } \\
\text { dinámicas durante las primeras sesiones sería bueno ya que la parte teórica } \\
\text { es aburrida. }\end{array}$ \\
\hline P15S12 & $\begin{array}{l}\text { Yo ya había tomado la clase en otra carrera, la cual fue muy teórica y me } \\
\text { agrado esta clase porque llevamos a la prásctica la metodología del ABP }\end{array}$ \\
\hline P15S2 & $\begin{array}{l}\text { La clase está bien fundamentada pero siento que se necesita hacer más } \\
\text { actividades colaborativas. }\end{array}$ \\
\hline P15S4 & $\begin{array}{l}\text { Me gusto la calse y no me gusta la tería especialmente el ABP y me } \\
\text { pregunté y esto que. Pero cuando ya hicimos el proyecto entendí para que } \\
\text { servía y cómo funcionaba y me gusto la clase. }\end{array}$ \\
\hline
\end{tabular}

Los sujetos están plenamente convencidos que mediante la investigación se gesta el conociemiento. Investigar no significa buscar fuentes de información, buscar hasta encotrar una intención, exige por lo tanto, contar con un plan de acción, es decir un programa de trabajo, donde se especifique la manera en la que van a realizar la investigación. Los hallazgos confirman lo mencionado por Paineán, Aliaga y Torres (2012).

\section{CONCLUSIONES}

El ABP establecido para trabajar en clase de diseño de proyectos, permitió hacer indagaciones, se presentaron resultados y se evaluaron conocimientos.

El ABP no es una metodología con la que estén acostumbrados a trabajar ni los docentes ni los estudiantes, ya que implica un cambio significativo en el concepto de enseñanzaaprendizaje desde una mirada activa como lo refiere López (2012) 
La metodología per se, mostró ser util en cuanto a la identificación del problema y el planteamiento de objetivos o metas para lograr resolver dicho problema donde entró en juego el pensamiento crítico y reflexivo

El trabajo en equipo y la discusión de resultados confirma que el aprendizaje requiere un ambiente físico con infraestructura apropiada y un ambiente afectivo respetuoso y asertivo tal como lo señala Barell (2001); por lo tanto, la eficacia en el desarrollo de la competencia investigativa esta presente en grupos reducidos.

Mediante el análisis de resultados se concluye que la participación incrementa y se comprende mejor lo que se esta planteado en las situaciones reales. Como lo dice la teoría constructivista desde el enfoque cognitivo y social (Schunk, 1997;Ausbel, 2006; Vygotsky, 1934; Coll, 1990; Pozo, 1999; Díaz y Hernández, 2002; Daniels, 2003; Hernández, 2006; Ponce, 2010, entre otros).

El aprendizaje se da de manera interna o cognitiva y de manera externa o social, en conjunto, dichos aprendizajes llevan a una mejor comprensión del mundo, del manejo de la información y de la adaptabilidad de los conocimientos para sortear problemas de cualquier índole a lo largo de la vida cotidiana y profesional.

\section{BIBLIOGRAFIA}

Barell, J. (2006). El aprendizaje basado en problemas, un enfoque investigativo. Argentina: Manantial

Barrows H., Tamblyn R. (1980)Problem-Based Learning: An Approach to Medical Education.[En línea] New York: Springer Publishing Company, Inc. Disponible en: http://books.google.com.mx/books?id=9u5DJuQq2UC\&pg=PA195\&dq=BARROWS+H.S.\&hl=es419\&sa=X\&ei=Y5WPUrLZNeTg2gXoxYGAAg\&ved=0CC0Q6AEwAA\#v=on epage \&q=BARROWS\%20H.S.\&f=false [Recuperado el 2013, 22 noviembre]

Cansigno, Y. (2006) El francés lengua de comunicación internacional. Reencuentro [en liena] No. 47. Disponible en: http://www.redalyc.org/articulo.oa?id=34004704 [2013, 22 de septiembre]

Delors J., (1996). La educación encierra un tesoro. Francia: UNESCO

Egido, I., Arana, R., Cerillo, R., De la Herrán, A., De Miguel, S., Gómez, M., Hernández, R., Izuzquiza, D., Murillo, F., Pérez,M. (2006) Aprendizaje basado en problemas. Estrategia metodológica y organizativa del currículum para la calidad de la 
enseñanza en los estudios del magisterio. Revista Interunivesitaria de Formación de Profesorado [en línea] Vol.20, No. 3. Disponible en: http://www.redalyc.org/articulo.oa?id=27411311007 [2013, 22 septiembre]

Escribano, A. y del Valle, A. (coords.) (2008). El aprendizaje basado en problemas. Una propuesta metodológica en educación superior. $2^{\mathrm{a}}$. Ed. España: Narcea.

López, M. (2008) El Aprendizaje basado en problemas. Una propuesta en el contexto de la educación superior en México. Tiempo de educar. [En línea] Vol. 9, No. 18.Disponible en: http://www.redalyc.org/articulo.oa?id=31111811003 [2013, 22 septiembre]

Márquez, V., Uribe, I., Montes, R, Monroy, C., Ruiz, D., Domínguez E. (2011) Satisfacción académica con el ABP en estudiantes de licenciatura de la universidad de Colima, México. Revista Intercontinental de Psicología y Educación [en línea] Vol. 13, No. 1. Disponible en: http://www.redalyc.org/articulo.oa?id=80218382003 [2013, 19 septiembre]

Marzano, R. (1997). Dimensiones del aprendizaje. Guadalajara: Iteso

Morales P., Landa V. (2004) Aprendizaje basado en problemas. Theoria [En línea] Vol. 13.

Disponible

en:

http://campus.usal.es/ ofeees/NUEVAS_METODOLOGIAS/ABP/13.pdf

[Recuperado el 2013, 22 noviembre]

Organización para la Cooperación y el Desarrollo, IMHE (2011).http://www.oecd.org/fr/sites/eduimhe/imheoecdforumonhighereducationlistofmemberinstitutions.htm

Paineán O., Aliaga V., Torres T. (2012) Aprendizaje basado en problemas: evaluación de una propuesta curricular para la formación inicial docente. Estudios Pedagógicos [en línea], Vol. XXXVIII, No. 1. Disponible en: http://www.scielo.cl/pdf/estped/v38n1/art10.pdf [2013, 29 de septiembre]

Rosler R., Zaloff, J., Hernández D., Torino R., Socolovsky M., González Abbat S. (2012) La insoportable pasividad de la clase teórica. Revista Argentina de Neurociencia [en línea] Vol. 22. Disponible en: http://www.scielo.org.ar/pdf/ranc/v22n2/v22n2a08.pdf [2013, 26 septiembre] 
Sanchez I. y Ramis F. (2004) Aprendizaje significativo basado en problemas. Horizontes Educacionales [en línea], No. 9. Disponible en: http://www.redalyc.org/articulo.oa?id=97917171011[2013, 22 de septiembre]

Sola, C. (dir.), (2005). Aprendizaje Basado en Problemas: de la teoría a la práctica. México: Trillas.

Secretaría de Educación Pública. (2008) Acuerdo 444 del Sistema Nacional de Bachillerato. [en línea] México: Diario Oficial de la Federación. Disponible en: http://www.cbachilleres.edu.mx/cb/comunidad/docentes/pdf/Reforma_curricular /Acuerdos/ACUERDOS_RIEM/Acuerdo444SNB.pdf 